\title{
MPP1 interacts with DOPC/SM/cholesterol in an artificial membrane system using Langmuir-Blodgett monolayer
}

\author{
Mohamed Elderdfi ${ }^{1}$, Jolanta Zegarlińska ${ }^{1}$, Walis Jones ${ }^{2}$ and Aleksander F. Sikorski ${ }^{1}$ \\ ${ }^{1}$ Department of Cytobiochemistry, Faculty of Biotechnology, University of Wrocław, 50-383 Wrocław, Poland \\ ${ }^{2}$ BioPharm Enterprises Limited, Tanyfron 65 Gwaun Afan, Cwmafan, Port Talbot, Neath Port Talbot Wales, United Kingdom
}

\begin{abstract}
The interaction between membrane palmitoylated protein-1 (MPP1) with lipid bi- and monolayers composed of a DOPC/SM/Chol mixture was investigated. MPP1 co-migrates with liposomes to the top of the liposome flotation gradient, indicating binding of MPP1 with liposomes. The injection of MPP1 into the subphase of a Langmuir-Blodgett (LB) monolayer of the above lipid composition induced an increase in surface pressure, indicating that MPP1 molecules were incorporated into the lipid monolayer. The compressibility modulus isotherms of MPP1, lipids and lipid-MPP1 films have essentially different shapes from one another. Pure MPP1 isotherms were characterized by a peak in surface pressure of $25-35 \mathrm{mNm}^{-1}$. This transition disappears in isotherms obtained with lipid monolayers in the presence of MPP1, which suggests an interaction between the protein and the lipid monolayers. In addition, this interaction is sensitive to the presence of cholesterol in the lipid monolayer, as the addition of MPP1 into the subphase of lipid monolayers containing cholesterol resulted in a much larger increase in surface area than when MPP1 is injected into the subphase of a lipid monolayer devoid of cholesterol. In conclusion, the data demonstrates that MPP1 interacts with lipid mixtures in two different model membrane systems.
\end{abstract}

Key words: MPP1 - Protein-lipid interactions - Flotation assay — Langmuir-Blodgett monolayer

\section{Introduction}

Membrane palmitoylated protein 1 (MPP1), is a member of a family of membrane-associated guanylate kinase homologue proteins (MAGUKs) and was originally identified as a membrane skeleton protein in erythrocytes (Dimitratos et al. 1999). Previous studies have confirmed that MPP1 anchors to the lipid bilayer in the erythrocyte membrane by constituting a ternary-complex with glycophorin $\mathrm{C}$ and protein 4.1 (Alloisio et al. 1993; Nunomura et al. 2000; Mburu et al. 2006). Human erythrocyte MPP1 consists of a single PDZ domain, a central SH3 domain, a C-terminal GUK domain and a D5 motif located between the SH3 and GUK domains (Fanning and Anderson 1996; Ruff et al. 1991; Seo et al. 2009). Recently, MPP1 was shown to play a crucial role in lateral membrane organization that may be involved in the molecular mechanism of a yet-unexplored haemolytic anaemia ( ach et al. 2012).

Correspondence to: Aleksander F. Sikorski, Department of Cytobiochemistry, Faculty of Biotechnology, University of Wrocław, 50-383, Wrocław, Poland

E-mail: aleksander.sikorski@uwr.edu.pl
However, the interaction of MPP1 with membrane proteins leading to resting-state raft-stabilization does not involve membrane skeleton proteins such as actin, since extraction and/or depolymerisation does not affect the amount of detergent-resistant membrane (DRM) and membrane-fluidity, as measured by FLIM using the di- 4 probe (Biernatowska et al. 2013; Łach et al. 2012; Podkalicka et al. 2015). On the other hand, our team has recently proposed that MPP1 interacts with flotillins in the native membrane and in DRMs.

The current view of the biological membrane is that lipids and proteins mutually interact in a dynamic but transient way to accomplish membrane functions. It has become clear now that lateral heterogeneity strongly influences our concepts of the structure of the lipid bilayer and that lipid and protein sorting is highly dependent on the lateral organization of the membrane (Engelman 2005). The enrichment of sphingomyelin and cholesterol in the membrane, in general, and in membrane raft domains, in particular, has been considered by several studies reporting on the roles of these lipids within the membrane, including the interactions between these two principal components (Collado et al. 2005; Coste et al. 2006; Devanathan et al. 2006; Frazier et al. 2007). 
Packing defects and lateral heterogeneity may facilitate a number of biological functions of the membrane. Therefore, it is relevant to understand the forces controlling the lateral ordering and diffusion of lipids and their basic physical chemistry. In brief, laterally-separated phases may be induced by enzymatic cleavage of lipids (Holopainen et al. 1998), temperature (Mouritsen 1991), surface electrostatic associations (Rytömaa and Kinnunen 1996), and lipid-lipid (Lehtonen et al. 1996; Söderlund et al. 1999) or lipid-protein (Mouritsen and Bloom 1984) interactions. The ternary mixture consisting of dioleoylphosphatidylcholine (DOPC), sphingomyelin (SM) and cholesterol (Chol) has been used as a membrane-mimicking model (Yuan et al. 2002; Kulma et al. 2010; Nyholm et al. 2011; Tsukamoto et al. 2014). It has been shown that this lipid mixture can imitate the phaseseparation of cell membranes (Bezlyepkina et al. 2013), which provides an experimental alternative to native plasma membranes containing major membrane components, including phospholipids, sphingolipids and cholesterol.

A growing number of studies describe membrane-mimicking models, including liposomes and Langmuir-Blodgett (LB) monolayers. In this study, we ask the question as to whether MPP1, as single protein component, has the capability to interact with lipid mono- or bilayers which exhibit a complex lateral heterogeneity (DOPC/SM/Chol, 1:1:1 molar ratio) in two separate model systems, namely a liposome-flotation assay using liposomes, or with a lipid monolayer prepared from the lipid mixtures at the air-water surface using the LB monolayer film technique. LB monolayer is a powerful technique that allows the formation of monolayer lipid films, together with the ability to introduce protein into the subphase buffer at a range of concentrations and under the desired physiologicallycompatible conditions including subphase buffer, $\mathrm{pH}$ and temperature. The results of this study provided direct evidence of such an interaction between MPP1 and lipid and suggest the possible participation of this binding mechanism in lateral organization of the membrane.

\section{Material and Methods}

Overexpression and purification of MPP1 in a bacterial system

The MPP1 protein construct was obtained by subcloning the MPP1 gene sequence (Sequence ID: NP_002427.1) into the pRSET A plasmid (Invitrogen) using BamHI and XhoI restriction enzymes (Promega). In order to express MPP1 protein, Escherichia coli BL21 were transfected with purified plasmid constructs. After expression of MPP1 in Escherichia coli BL21 cells, using isopropyl $\beta$-D-1-thiogalactopyranoside (IPTG) as an inducer for 16 hours at $18^{\circ} \mathrm{C}$, the recombinant protein with a His6-tag at the amino terminus was extracted with $8 \mathrm{M}$ urea in $20 \mathrm{mM}$ Tris- $\mathrm{HCl}$ and $150 \mathrm{mM} \mathrm{NaCl}$ at $\mathrm{pH} 8$ and affinity-purified on an immobilized Ni-NTA-affinity resin (Qiagen) on an Econo-Pac ${ }^{\oplus} 10$ DG chromatography column (Bio-Rad Laboratories). Lowering the temperature to $18^{\circ} \mathrm{C}$ after bacterial induction was chosen to minimize the formation of inclusion bodies and to improve protein solubility.

The purified protein was then analysed using SDS polyacrylamide gel electrophoresis with a Coomassie blue stain. The concentration of MPP1 was calculated using an absorbancy coefficient at $280 \mathrm{~nm}$ calculated using the ExPASy ProtParam program (Wilkins et al. 1999). MPP1 was centrifuged at 10,000 rpm to remove any precipitated material and to ensure the homogeneity of the sample before use. Circular dichroism measurements of the proteins were performed after dialysis on a JASCO J-815 (Spectroscopic Co. Ltd, Japan). The spectra were measured from $20-70^{\circ} \mathrm{C}$ at $0.2 \mathrm{~nm}$ resolution from 190 to $240 \mathrm{~nm}$ in Tris- $\mathrm{HCl}$ buffer containing $5 \mathrm{mM}$ Tris- $\mathrm{HCl}, 50 \mathrm{mM} \mathrm{NaCl}$ (pH 7.4).

\section{Lipids}

Sphingomyelin (egg), 1,2-dioleoyl-sn-glycero-3-phosphocholine (DOPC) and 1,2-dioleoyl-sn-glycero-3-phosphoserine (DOPS) were purchased from Avanti Polar Lipids and Cholesterol (Chol) was obtained from Northern Lipids. Lipid concentrations were quantified by phosphorus analysis (Rouser et al. 1966). Cholesterol concentration was quantified using the Cholesterol Kit (BioSystems).

\section{Flotation assay}

Liposomes were prepared by the hydration of a dry lipid film (Bangham et al. 1965; Morton et al. 2012). Briefly, chloroform solutions of the individual lipids, namely, DOPC, SM and cholesterol, were mixed at a 1:1:1 molar ratio in a roundbottom flask and the chloroform evaporated in a nitrogen stream to obtain a thin lipid film. The film was then further dried in a vacuum desiccator for at least 2 hours, or overnight. The lipids were resuspended in Hepes-buffered saline (HBS buffer) (20 mM HEPES, $150 \mathrm{mM} \mathrm{NaCl}, \mathrm{pH}$ 7.4). The hydrated liposomal suspension was subsequently extruded through a $0.4 \mu \mathrm{m}$ polycarbonate membrane filter at a temperature of $64^{\circ} \mathrm{C}$ under high pressure gaseous nitrogen. The filter was then sequentially replaced by $0.2 \mu \mathrm{m}$ and $0.1 \mu \mathrm{m}$-pore membranes, and the extrusion cycles were repeated 10 times independently for each filter. The size of the liposomes was determined using a ZetaSizer (Malvern). Liposomes were stored at $4^{\circ} \mathrm{C}$ until use.

MPP1 at concentrations of 50 and $150 \mathrm{nM}$ were prepared with $0.4 \mathrm{mg} / \mathrm{ml}$ lipid-liposomes in HBS buffer to a final volume of $250 \mu \mathrm{l}$ and incubated at room temperature for 30 minutes. Control samples contained MPP1 at the same concentrations, but without liposomes. After incubation, samples were transferred to ultracentrifuge tubes and mixed with $250 \mu \mathrm{l}$ of $60 \%$ sucrose by pipetting up and down few times. The 
samples were carefully overlaid with $0.8 \mathrm{ml}$ of $15 \%$ sucrose, followed by $1.8 \mathrm{ml}$ of $10 \%$ sucrose in HBS buffer, and finally with $1.0 \mathrm{ml}$ of HBS buffer without mixing, so as to establish a sucrose gradient. The samples were then centrifuged at $\sim 200000 \times g(45000 \mathrm{rpm}, 60 \mathrm{Ti}$ rotor $)$ for 2 hours at $4^{\circ} \mathrm{C}$. After centrifugation, 6 fractions were taken, starting from the top of gradient and, after addition of SDS to a final concentration of $1 \%$, fractions were analyzed via dot-blot assay. Equal volumes of samples were loaded into the wells of a dot-blotter (Hoefer Scientific Instruments) and transferred onto a nitrocellulose membrane by vacuum filtration after which the membrane was blocked for 1 hour at room temperature. The samples were incubated with primary mouse monoclonal anti-MPP1 antibodies (Abnova, 1:1000) for 3 hours at room temperature or, alternatively, overnight at $4^{\circ} \mathrm{C}$. Membranes were washed three times for $5 \mathrm{~min}$ in Tris-buffered saline with Tween 20 (TBS-T) (20 mM Tris-HCl, $150 \mathrm{mM} \mathrm{NaCl,} \mathrm{0.05 \%} \mathrm{Tween,}$ pH 7.4). Secondary goat anti-rabbit antibodies (Santa Cruz 1:10000) were then added and incubated for 1 hour at room temperature. Enhanced chemiluminescence (ECL) was used for detection and viewed using ultraviolet products (UVP) Bio Spectrum Imaging System (United Kingdom).

\section{Langmuir-Blodgett monolayer}

Monolayer experiments were carried out as described previously by Grzybek et al. (2009). The measurements were performed using a $70 \mathrm{~cm}^{2}$ teflon Langmuir trough connected with motorized barriers (Nima Technology) equipped with a Nima tensiometer ST 9000 (Nima Technology) along with a filter-paper Wilhelmy-plate (KSV Nima, Biolin Scientific) to measure surface pressure. The trough was placed in an enclosed chamber, facilitating flushing with a nitrogen stream and temperature was controlled by a water jacket (Julabo F12, Germany) to $22^{\circ} \mathrm{C}$. A subphase buffer $(60 \mathrm{ml})$, containing $5 \mathrm{mM}$ Tris- $\mathrm{HCl}, 150 \mathrm{mM} \mathrm{NaCl}, 0.5 \mathrm{mM}$ EDTA, $0.5 \mathrm{mM} \mathrm{DTT}$ and $\mathrm{pH} 7.4$ was used. Monolayers were formed by injecting a chloroform solution of a lipid-mixture with a Hamilton syringe on the surface of the subphase buffer. After spreading, the solution was left for 10 min to allow for solvent evaporation and a plot of the surface pressure (П) versus molecular area $(\mathrm{A})$ was plotted. For each experimental condition, at least 3 independent monolayers were prepared and, for each lipid mixture monolayer, 4-5 isotherms were recorded without reaching the collapse pressure, prior to adding any protein. When similar lipid isotherms were obtained, the protein was then injected. Aliquots, $600 \mu \mathrm{l}$, of MPP1 previously dialyzed against the subphase buffer were injected into the subphase. The surface pressure against the area was recorded after stirring the subphase for 4 minutes using a small stirrer bar, followed by another 4 minutes of stabilization. For analysis, the most consistent isotherms from each monolayer were chosen. Independent and dependent variables collected by the instrument were imported into Microsoft Excel ${ }^{\circledast}$ and the surface compressibility modulus $\left(\mathrm{Cs}^{-1}\right)$ of the monolayer was calculated from the first derivative of the monolayer surface pressure and area per molecule data using the following formula (Gicquaud et al. 2003):

$\mathrm{Cs}^{-1}=-\mathrm{A} \times(\Delta \Pi / \Pi \mathrm{A})$

where $\mathrm{A}$ is the area per molecule at the indicated surface pressure and $\Pi$ is the corresponding surface pressure.

GraphPad PRISM ${ }^{\circledast} 6$ Software was used for Scatchard analysis, using a nonlinear regression curve fit for one-site binding. The results calculated by GraphPad display the best-fit values for the binding parameter, i.e. equilibrium dissociation constant $\left(\mathrm{K}_{\mathrm{D}}\right)$. Standard deviation and Student's $t$-test were used to assess the variability of obtained data applying MS Excel ${ }^{\oplus}$ procedures.

\section{Results}

\section{Liposome flotation assays}

A Coomassie Blue stained SDS-10\% PAGE electropherogram of purified recombinant MPP1 is shown in Figure 1A. Binding of MPP1 to liposome membrane was assessed using liposomes composed of a DOPC, SM and

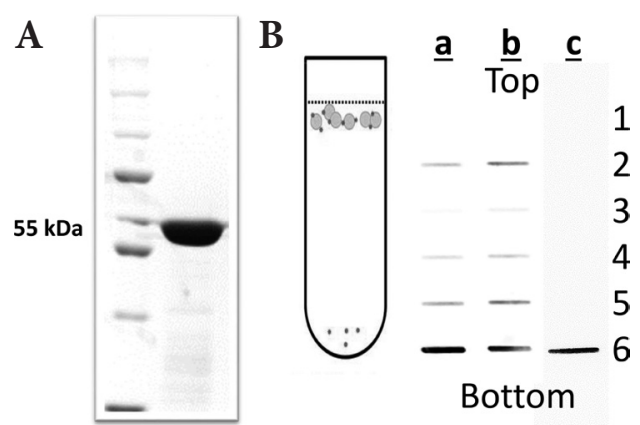

Figure 1. Interaction of MPP1 with liposomes. A. MPP1 was analysed by SDS-10\% PAGE, Coomassie staining and size-calibrated using standard molecular weight markers. B. Interaction of MPP1 with liposomes composed of DOPC/SM/Chol at a molar ratio of 1:1:1. Flotation assay of recombinant MPP1 added to liposomes $(0.4 \mathrm{mg} / \mathrm{ml}$ and $100-\mathrm{nm}$ average diameter). Fractions (1-6) were collected from the top to the bottom of the centrifuge tube and MPP1 in each fraction was analysed by Dot-blot assay with mouse monoclonal anti-MPP1 antibodies. Flotation of MPP1 depends on the binding of MPP1 to the liposomes. Lanes a and b: $50 \mathrm{nM}$ and $150 \mathrm{nM}$ MPP1, respectively, with liposomes; MPP1 co-migrates with liposomes to the top of the gradient. Lane c: $150 \mathrm{nM}$ MPP1 is found at the bottom of the gradient in the absence of liposomes. 
cholesterol (1:1:1 molar ratio) mixture. MPP1 was incubated with liposomes at room temperature for 30 minutes. Next, an equal volume of dense sucrose solution was added and the final mixture was placed at the bottom of a sucrose gradient and overlayered with a series of lower- density solutions and then ultra-centrifuged (see Materials and Methods). If MPP1 binds to liposomes, it will float towards the top of the centrifugation tube in association with the liposomes. An example of the dot-blot assay of gradient fractions of liposome mixtures containing 50
A

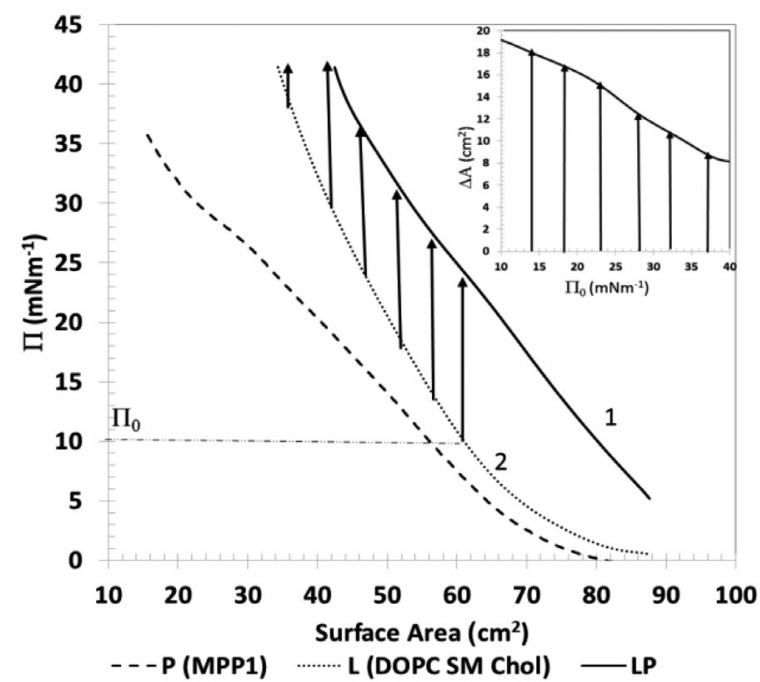

C

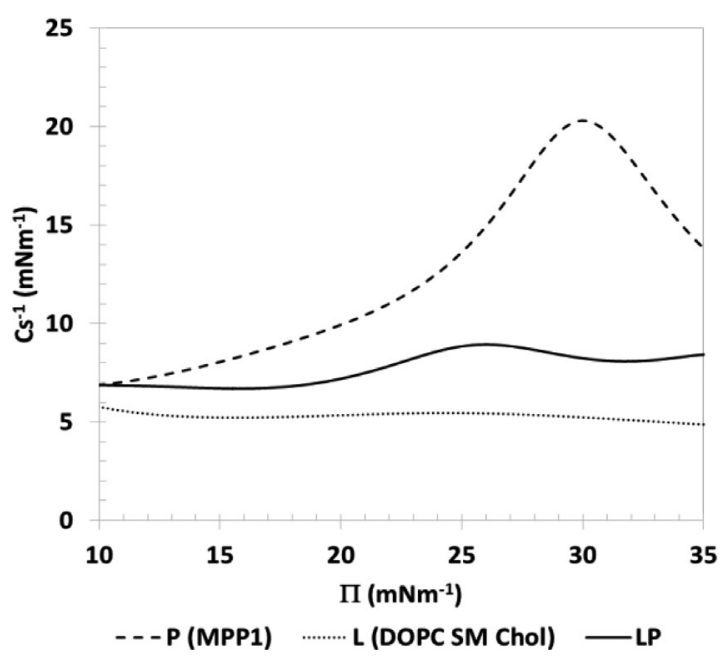

B

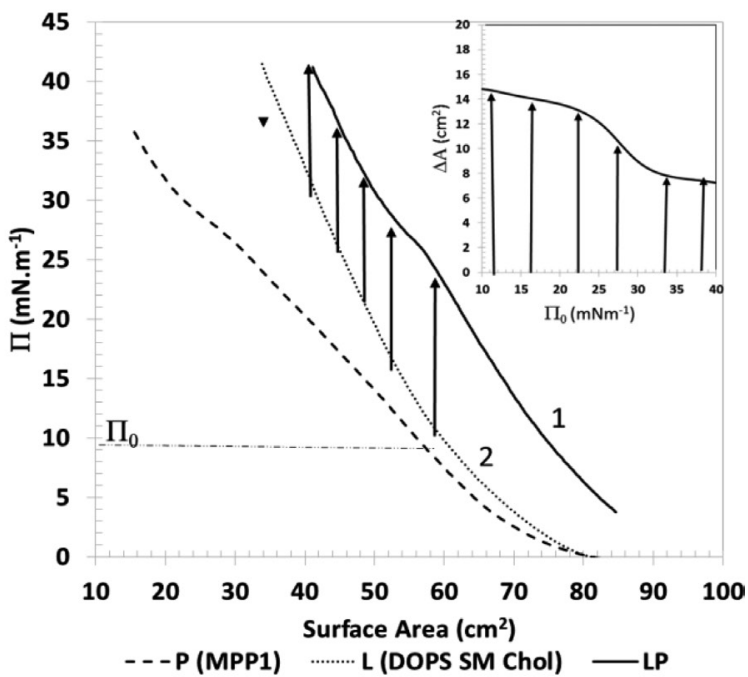

D

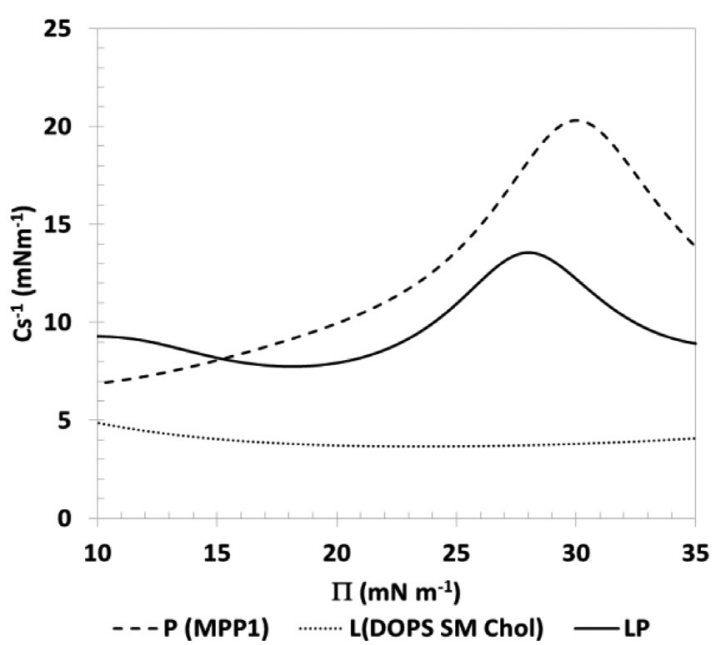

Figure 2. Surface Pressure-Area Isotherms of MPP1 with lipid monolayers. The compression isotherms for the lipid monolayers (DOPC/ SM/Chol (A); DOPS/SM/Chol (B)) alone (dotted curve), MPP1 alone (dashed curve), and lipid monolayers in the presence of $10 \mathrm{nM}$ MPP1 in the subphase (solid curve). The compressibility modulus, $\mathrm{Cs}^{-1}-\Pi$, was calculated as a function of the surface pressure of the films from the corresponding П-A isotherms (DOPC/SM/Chol (C); DOPS/SM/Chol (D)). The molar ratio of the lipids in the monolayers was 1:1:1. Insets: The dependence of $\triangle \mathrm{A}$ on $\Pi_{0}$ represents the MPP1 contribution in the monolayer, calculated as the difference between the MPP1-lipid monolayer isotherms (curve 1) and lipid monolayers alone (curve 2) at the same surface pressure. $\Pi_{0}$, surface pressure; $\triangle \mathrm{A}$, an increment of the surface area from the lipid monolayer without MPP1 to the same lipid monolayer after MPP1 injection at the same surface pressure. In this case, the surface area is a relative value, because the area per molecule of MPP1 cannot be calculated, as the size of MPP1 is undetermined. 
and $150 \mathrm{nM}$ MPP1 is shown in Figure 1B, lanes a and b. Lane $\mathrm{c}$ shows a result of a dot-blot assay of a 150 nM MPP1 sample in which the liposome suspension was omitted. When the incubation mixture contained both liposomes and MPP1 (Fig.1Bab), a reasonable fraction of MPP1 is found in the top fraction from the density gradient, while in the absence of liposomes it is found only in the bottom fraction. These results indicate that MPP1 interacts with liposomes.

\section{Langmuir-Blodgett monolayer study}

\section{Surface pressure-area isotherms}

To gain a more detailed insight into possible MPP1-lipid interactions, we investigated the effect of MPP1 on LB lipid monolayers composed of 1:1:1 molar ratios of a DOPC/ $\mathrm{SM} /$ Chol mixture. In a series of experiments, a lipid monolayer was formed and MPP1 was then injected into the subphase buffer beneath the lipid monolayer. Figure $2 \mathrm{~A}$ shows the surface pressure-area isotherms of the lipids alone (dotted curve) or with MPP1 present in the subphase (solid curve), and the isotherms of pure MPP1 without lipids as a control (dashed curve). The surface pressure of the lipid isotherms alone increases to a maximum value at $\sim 41 \mathrm{mNm}^{-1}$ as the surface area is reduced during compression. The addition of MPP1 in the subphase modifies the behavior of the isotherms. The isotherms show that, when MPP1 is present in the subphase of a lipid monolayer, the recorded initial surface pressure is higher than that of the lipid monolayer alone and protein alone. Moreover, at any given pressure, the addition of MPP1 significantly increased the pressure of the lipid monolayer. The complex features of the surface pressure-area isotherms of the obtained isotherms can be better shown by plotting the $\mathrm{Cs}^{-1}$ (Gicquaud et al. 2003). The pure MPP1 isotherms are characterized by a peak that implies an apparent change in the protein conformation at the air-liquid interface at a surface pressure of $25-35 \mathrm{mNm}^{-1}$. It is interesting that this transition at $25-35 \mathrm{mNm}^{-1}$ disappears in the isotherms obtained in the presence of a lipid monolayer, suggesting that MPP1 incorporation into lipid monolayer results in a restructuring of the lipid/protein packing at the surface (Fig. 2C). The inset in Figure 2A shows the area increment, $\triangle \mathrm{A}$, resulting from MPP1 incorporation into the lipid monolayer from the subphase. By progressively restricting the monolayer area and increasing the surface pressure, this effect $(\Delta \mathrm{A})$ decreased but did not totally disappear. This means that the area of the lipid-MPP1 monolayer in the presence of the protein in the range of the physiological pressures, $>30 \mathrm{mNm}^{-1}$, is larger than the area of lipid monolayers in the absence of protein (inset Fig. 2A).

It was interesting to compare the above described interaction with the interaction of MPP1 with a lipid monolayer containing charged lipid, i.e. one in which DOPC was substituted by DOPS. The $\Pi$-A and $\mathrm{Cs}^{-1}-\Pi$ isotherms of the DOPS/ SM/Chol lipid mixture show that, when MPP1 is injected into the subphase, the compression isotherms are characterized by a plateau ( $-\mathrm{A})$, or a peak $\left(\mathrm{Cs}^{-1}-\Pi\right)$, at the region corresponding to a surface pressure of $23-35 \mathrm{mNm}^{-1}$ (Fig. $2 \mathrm{~B}$ and $2 \mathrm{D})$. In the case of the DOPS-containing monolayers, the plateau ( $-\mathrm{A})$ or peak $\left(\mathrm{Cs}^{-1}-\Pi\right)$ does not disappear in the presence of MPP1, in contrast to the DOPC-containing monolayers. It is interesting that the surface pressure range at which the plateau of $\Pi-\mathrm{A}$ or peak of $\mathrm{Cs}^{-1}-\Pi$ curves are similar to those obtained for pure MPP1 (dashed lines) and the penetration of the protein into the air-buffer interface

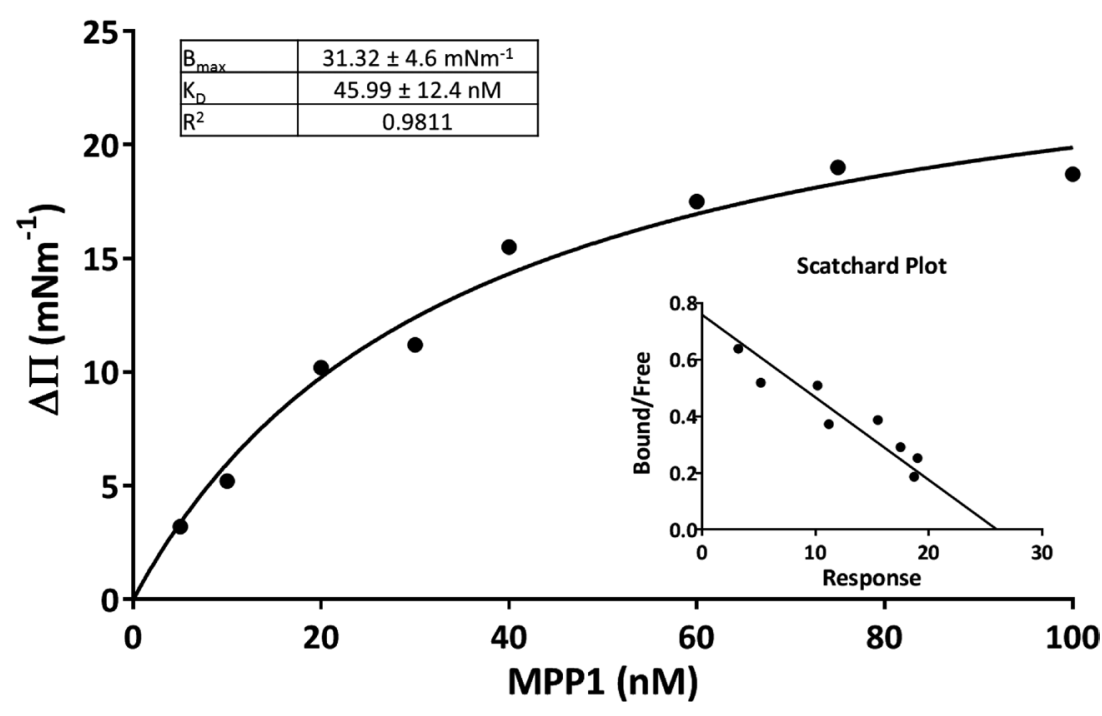

Figure 3. The effect of MPP1concentration in the subphase on the initial surface pressure change $(\Delta \Pi)$ of the monolayer. Scatchard plot depicting the analysis of the curve data. MPP1 was injected into the subphase beneath a monolayer of DOPC/SM/Chol (1:1:1) at $22^{\circ} \mathrm{C}$. One-site binding was used to fit MPP1binding with a monolayer. Inset table presents a summary of results obtained from the nonlinear regression fit. $B_{\max }$, maximal change in surface pressure, $\mathrm{K}_{\mathrm{D}}$, equilibrium dissociation constant. Error values, standard error of fit. 
dramatically decreased at this surface pressure range (inset Fig. 2B). These results indicate that the behavior of the protein at the air-liquid interface is also dependent upon the composition of the lipid monolayer.

Effect of the initial MPP1 concentration in the subphase on monolayer surface pressure

The increase of surface pressure $(\Delta \Pi)$ of the DOPC/SM/Chol (1:1:1) monolayer after injection of MPP1 into the subphase was monitored and plotted as a function of concentration, and a hyperbolic curve was obtained (Fig. 3). To estimate the apparent equilibrium, $\mathrm{K}_{\mathrm{D}}$, for the MPP1-lipid interaction, this data was fitted to a nonlinear regression fit using GraphPad PRISM 6 software (Fig. 3). The apparent $\mathrm{K}_{\mathrm{D}}$ determined by this method was $34.87 \pm 6.6 \mathrm{nM}$, and $\mathrm{B}_{\max }$ which is the maximal change in the surface pressure, was $26.8 \pm 2.0 \mathrm{mNm}^{-1}$. The data from the Scatchard plot showed a straight line, which implies a one-site binding interaction (inset Fig. 3).
Characteristics of surface pressure-area isotherms of DOPC/SM/Chol with different MPP1 concentrations in the subphase

Figure 4A shows the $\Pi-\mathrm{A}$ isotherms of MPP1-lipid monolayers consisting of DOPC/SM/Chol with various concentrations of MPP1, in the range of 5 to $40 \mathrm{nM}$, in the subphase. The initial surface pressure of the lipid monolayer increases with increasing MPP1 concentration in the subphase. This indicates that MPP1 molecules are incorporated into the lipid monolayer from the subphase. Above the above-mentioned transition that occurs at a surface pressure of $25-35 \mathrm{mNm}^{-1}$, the isotherms of DOPC/ $\mathrm{SM} /$ Chol monolayer when the subphase contains $\geq 20 \mathrm{nM}$ MPP1 approach and overlap each other. The $\mathrm{Cs}^{-1}-\Pi$ curves (Fig. 4C) show that, when the MPP1 concentration in the DOPC/SM/Chol monolayer subphase exceeds $20 \mathrm{nM}$, the transition observed at $25-35 \mathrm{mNm}^{-1}$ (Fig. 2) starts to be more pronounced than that observed for the MPP1 concentrations $\leq 20 \mathrm{nM} \mathrm{MPP} 1$ (Fig. 4C).
A

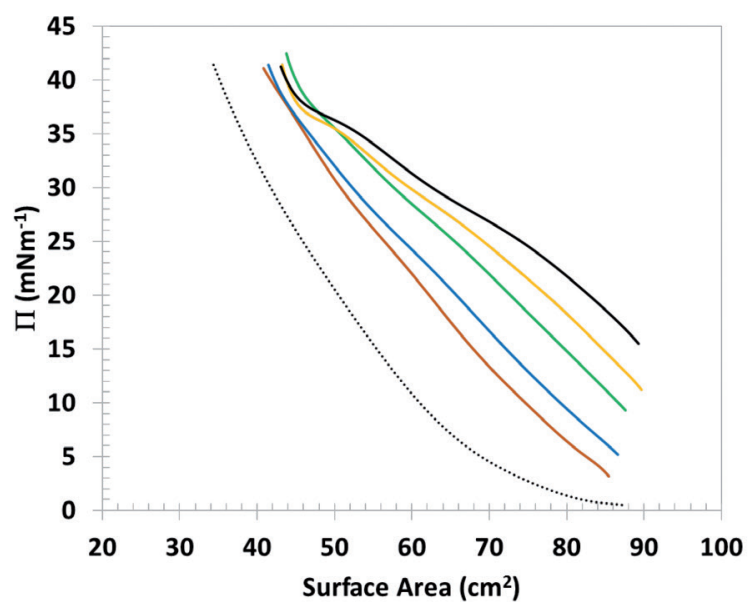

C

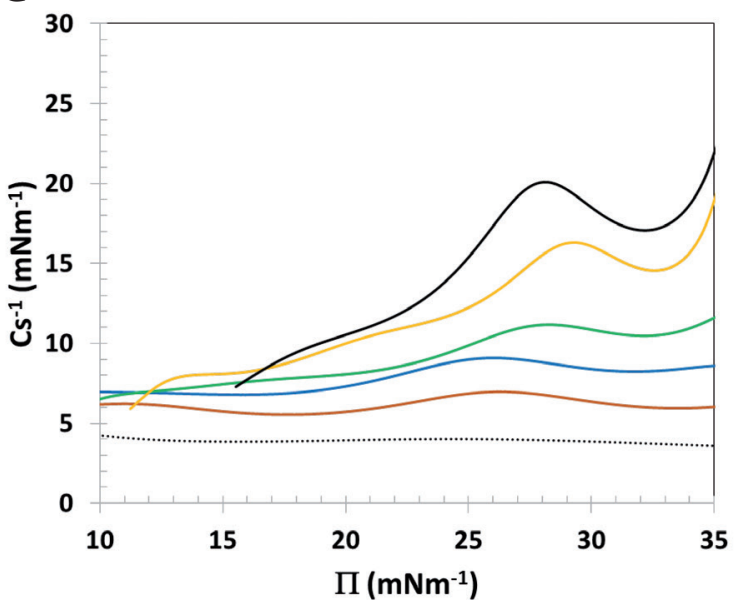

B

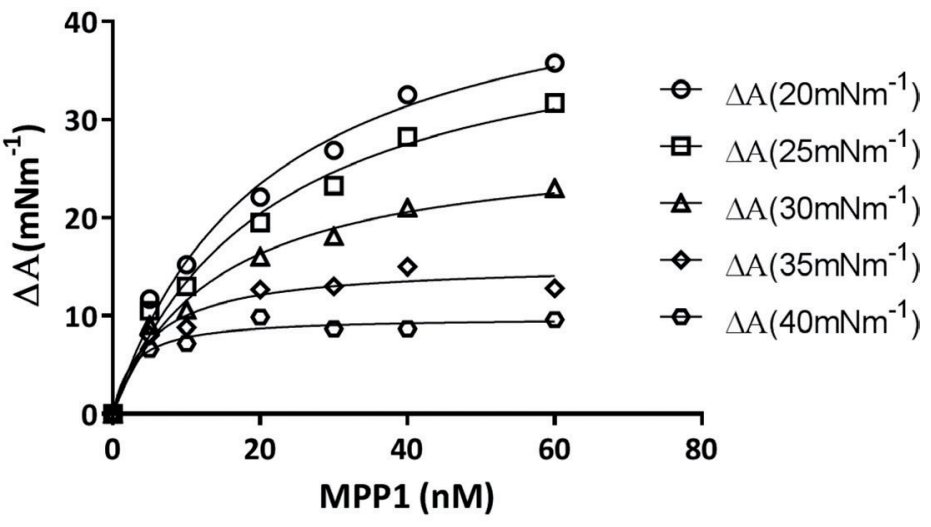

Figure 4. MPP1-lipid isotherms using a DOPC/SM/Chol lipid mix with different concentrations of MPP1 in the subphase. A. The $\Pi-\mathrm{A}$ isotherms of MPP1 - lipid monolayers in the presence of different MPP1 concentrations in the subphase. B. The change in area of MPP1-lipid monolayers versus MPP1 concentration. C. $\mathrm{Cs}^{-1}-\Pi$ isotherms calculated from $\Pi-\mathrm{A}$ isotherms shown in A. DOPC/SM/Chol isotherms at different MPP1 concentrations: brown line, $5 \mathrm{nM}$, blue line, $10 \mathrm{nM}$, green line, $20 \mathrm{nM}$, yellow line, $30 \mathrm{nM}$, black line, $40 \mathrm{nM}$, and dotted black line, the lipid monolayer without MPP1. 
To further characterize MPP1-lipid monolayer interactions, the changes in surface area of MPP1-lipid monolayers at surface pressures of $20,25,30,35$ and $40 \mathrm{mNm}^{-1}$ were plotted against MPP1 concentration (Fig. 4B). At a surface pressure $<35 \mathrm{mNm}^{-1}$, the change in the area $(\Delta \mathrm{A})$ increases with increasing protein concentration. On the other hand, at surface pressures of 35 and $40 \mathrm{mNm}^{-1}$, there is increasing change up to a protein concentration of $20 \mathrm{nM}$, whereas $\triangle \mathrm{A}$ of the lipid monolayers containing $\geq 20 \mathrm{nM}$ MPP1 was concentration-independent. When the points were fitted into a hyperbola, the $\mathrm{K}_{\mathrm{D}}$ values were in the range of $2.6 \mathrm{nM}$ for $40 \mathrm{mNm}^{-1}$ to $20.5 \mathrm{nM}$ for $20 \mathrm{mNm}^{-1}$, being relatively stable at various concentrations of MPP1 at various surface pressures. Moreover, as the $\mathrm{Cs}^{-1}$ - $\Pi$ dependence indicates, the monolayer in this MPP1 concentration range (30-40 nM) and $\Pi$ range $\left(25-30 \mathrm{mNm}^{-1}\right)$ resembles the characteristics of pure MPP1 at the air-water interface (compare Fig. 2B and $4 \mathrm{C}$ ). A possible explanation for this effect is proposed in the "Discussion".

\section{Effect of cholesterol on the interaction of MPP1 with lipid membranes}

To test whether the presence of cholesterol has an effect on the interaction of MPP1 with lipid monolayers, changes in the surface area $(\triangle \mathrm{A})$ of the DOPC/SM/Chol 1:1:1 monolayer and DOPC/SM 2:1 monolayer were measured after the addition of $20 \mathrm{nM} \mathrm{MPP} 1$ into the subphase (Fig. 5). The presence of cholesterol in the DOPC/SM monolayer facilitates the penetration of the monolayer by MPP1. Namely, the injection of $20 \mathrm{nM}$ MPP1 into the subphase resulted in a larger increase in $\Delta \mathrm{A}$ values in presence of cholesterol, compared to those observed in the absence of cholesterol. Moreover, at higher surface pressure, the difference becomes greater reaching at least a three-fold increase in the $\Delta \mathrm{A}$ values at a surface pressure of $35 \mathrm{mNm}^{-1}$ when cholesterol is present in the monolayer compared to the monolayer without cholesterol (Fig. 5). When the Student's $t$-test was applied in the comparison of the data obtained for DOPC/ $\mathrm{SM} / \mathrm{Chol} v s$ DOPC/SM monolayers, the differences observed at all surface pressure values appeared statistically significant $(0.0001<p<0.002)$. The data presented above indicates rather a strong dependence of the MPP1-lipid monolayer interaction on cholesterol.

\section{Discussion}

Previous studies involving MPP1 have mainly focused on protein-protein interactions, rather than binding of MPP1 to the membrane lipid bilayer. Here, we investigated the interaction between MPP1 and a lipid mono- or bilayer composed of DOPC, SM and cholesterol at a molar ratio of 1:1:1 in two separate model systems, namely a liposome-flotation assay and a LB monolayer film technique. The qualitative results obtained from the flotation assay showed that a fraction of MPP1 molecules was associated with the liposome fraction from the top of the sucrose gradient, indicating that MPP1 binds to the liposomes. In addition, separate in silico binding analyses by molecular modeling have predicted the ability of MPP1 to form homodimers, via domains on MPP1 that can bind to each other, such as the $\mathrm{SH} 3+\mathrm{HOOK}$ domain and an end-fragment of MPP1 that contains the GUK domain (Gosens et al. 2007). Formation of the MPP1 homodimer could be responsible for limiting the MPP1-lipid interaction.

More quantitative evidence of the interaction of MPP1 with lipids was also demonstrated via the LB method with lipid monolayer films. The LB monolayer method provides an insight into whether the interaction between the protein and lipids affects the properties of the lipid monolayer. The increase in the surface pressure of the lipid monolayer composed of the DOPC/SM/Chol mixture after injection of MPP1 into the subphase indicates that MPP1 molecules penetrate into the lipid monolayer (Fig. 2). This increase in the initial pressure is similar to that obtained by cytochrome c interaction with a cardiolipin phospholipid monolayer (Marchenkova et al. 2015), as well as to that observed for the interaction of a synthetic antimicrobial peptide, called V4, with both POPG and POPC monolayers (Yu et al. 2009).

The observed effect was dependent upon the concentration of the protein in the subphase (Figs. 3 and 4).

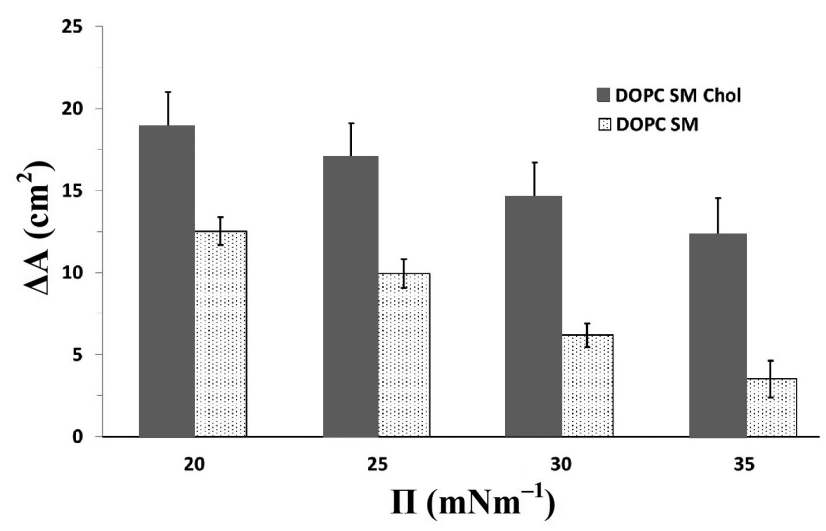

Figure 5. Effect of cholesterol on the interaction of MPP1 with lipid monolayers. The change in the surface area, $\Delta \mathrm{A}$, induced by MPP1 at different surface pressure values in lipid monolayers with and without cholesterol. The difference between the surface-area recorded for a lipid film in the presence of MPP1 in the subphase and for the corresponding film of lipid recorded in the absence of MPP1 as a function of the surface area available in the trough. The concentration of MPP1 in the subphase was $20 \mathrm{nM}$. DOPC/SM/ Chol (black column) and DOPC/SM (white dotted bars). Data represent the mean \pm S.D. For all pairs (DOPC/SM/Chol $v$ s. DOPC/ $\mathrm{SM}), p<0.002(n=4)$. 
However, when DOPC in the lipid monolayer film is substituted with DOPS, a negatively charged phospholipid, the properties of the monolayer seem less affected by MPP1, as the $\mathrm{Cs}^{-1}-\Pi$ isotherms display a well-pronounced peak at a $\Pi$ range of $25-30 \mathrm{mNm}^{-1}$ (Fig. 2), whereas in case of the DOPC lipid mixture, no peak was observed in the $\mathrm{Cs}^{-1}-\Pi$ isotherms. This may indicate a rather smaller fraction of MPP1 bound to or forming a common phase with the DOPS/SM/Chol monolayer. Therefore, the nature of the

A

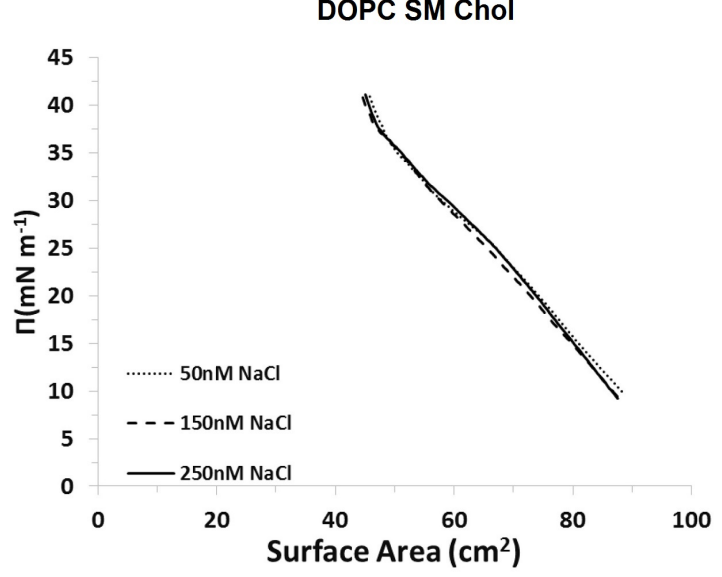

C

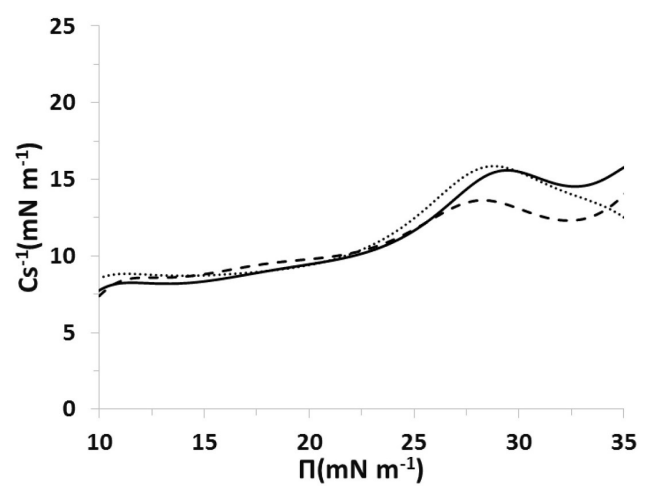

$\mathbf{E}$

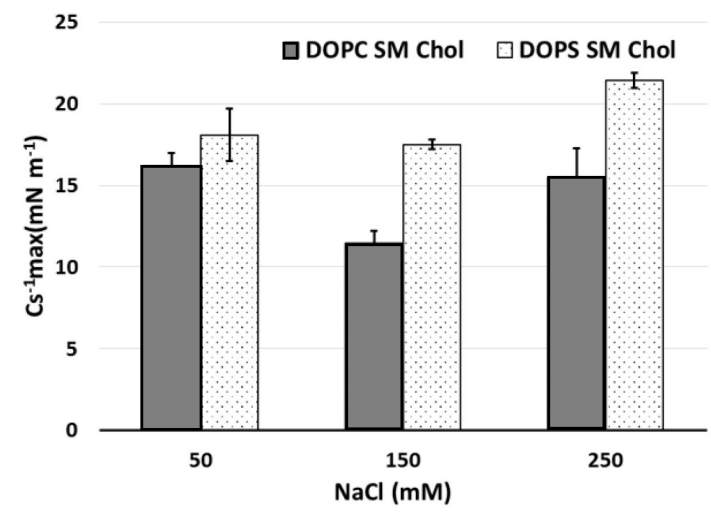

polar lipid head-groups could also play a role in MPP1lipid interaction.

The presence of lipid monolayers at the buffer surface enhanced the attraction of MPP1 to the interface, since such monolayers were immediately modified in the presence of $5 \mathrm{nM}$ MPP1, as shown by the immediate $\Delta \Pi$ increase upon addition of protein (Figs. 3 and 4). The $\mathrm{DOPC} / \mathrm{SM} / \mathrm{Chol}$ isotherms containing $\leq 30 \mathrm{nM} \mathrm{MPP} 1$ are also characterized by a transition at $25-35 \mathrm{mNm}^{-1}$

B

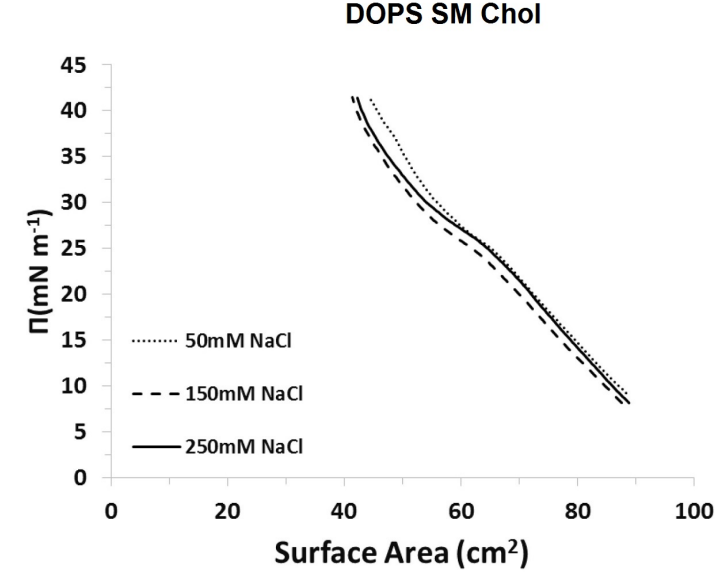

D

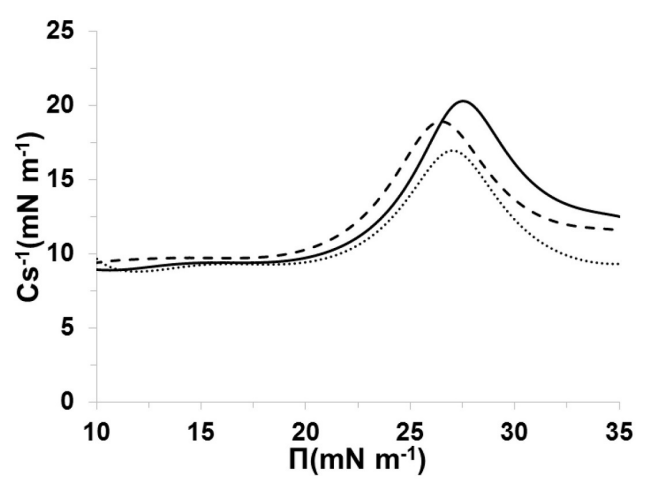

Figure 6. The effect of $\mathrm{NaCl}$ on the phase behavior of MPP1-lipid monolayers. The compression of MPP1-lipid isotherms in the presence of $50 \mathrm{mM}$ (dotted curve), $150 \mathrm{mM}$ (dashed curve), and $250 \mathrm{mM} \mathrm{NaCl}$ in the subphase (solid curve) (DOPC/SM/Chol (A); DOPS/SM/Chol (B)). The compressibility modulus, $\mathrm{Cs}^{-1}-\Pi$ isotherms was calculated from the corresponding $\Pi-\mathrm{A}$ isotherms as a function of the surface pressure of the films (DOPC/SM/Chol (C); DOPS/SM/Chol (D)). E. Maximum compressibility modulus, $\mathrm{Cs}^{-1}$ max , values, as a function of salt concentration for the MPP1lipid mixtures. DOPC/SM/Chol (black column) and DOPS/SM/ $\mathrm{Chol}$ (white dotted bars). The surface area is a relative value for the reason mentioned in the legend for Figure 2. Data represents the mean \pm S.D. $(n=3)$. 
(Fig. 4). Moreover, as the $\mathrm{Cs}^{-1}-\Pi$ dependence indicates, the isotherms of the lipid monolayer in the presence of this MPP 1 concentration range and $\Pi$ range resembles the characteristics of pure MPP1 protein at the air-buffer interface (compare Figs. 2C and 4C). This effect could be a result of a partial, transitory separation of the "protein domain" (phase) from the lipid-protein domain which is followed by the squeezing out of the protein from the monolayer at a surface pressure of around $35-40 \mathrm{mNm}^{-1}$

A

DOPC SM Chol

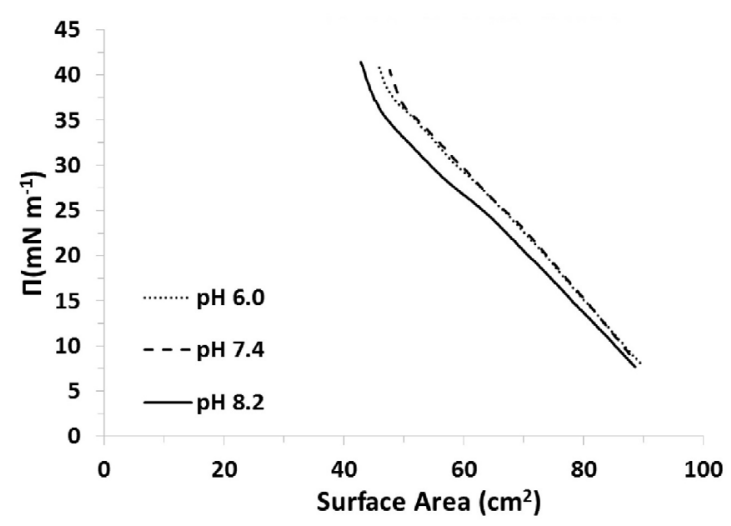

C

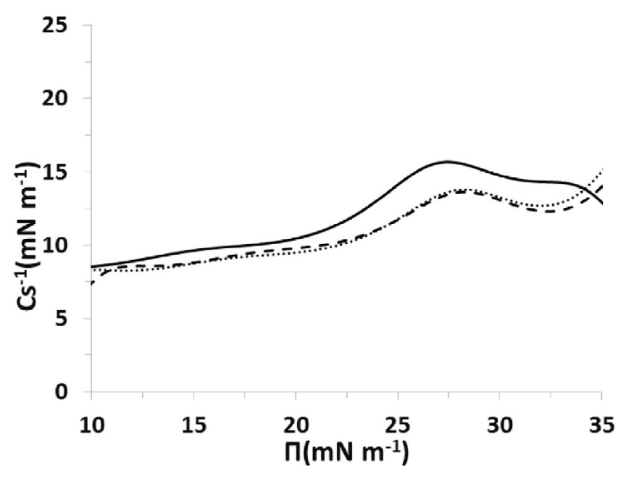

E

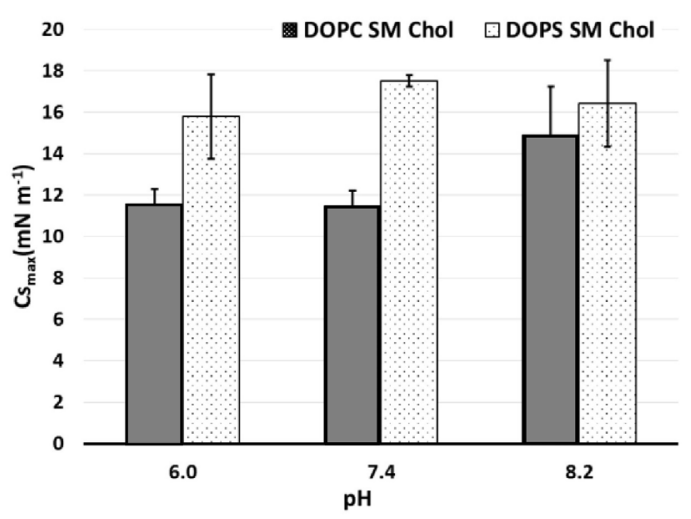

(compare Figs. 4A and 4C). The saturation observed in the increase in area in the higher $\Pi$ range could be due to the exchange of lipid molecules for MPP1 between the monolayer and the interphase and the binding of these lipid molecules by "interphase" MPP1. It should be noted that a certain amount of the protein remains tightly bound to the monolayer as squeezing out of protein at high surface pressure $\left(>35 \mathrm{mNm}^{-1}\right)$ does not result in any overlapping of such isotherms with the isotherms of the pure lipid

B

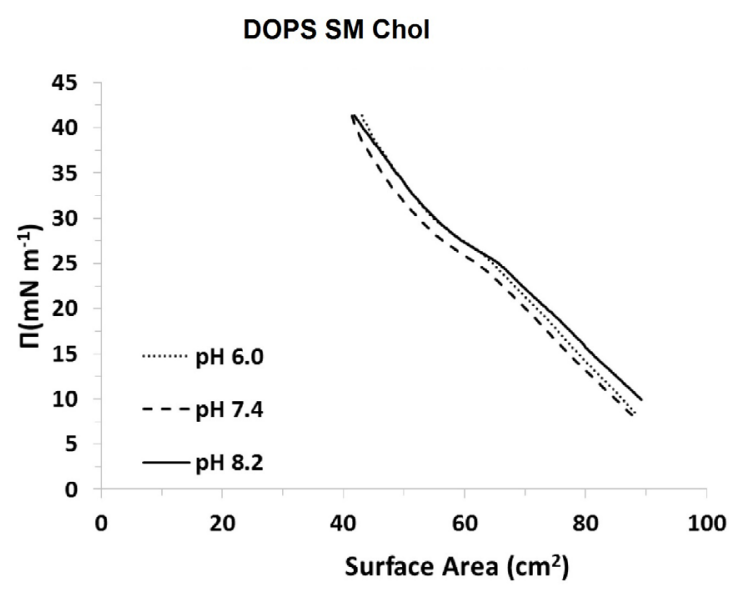

D

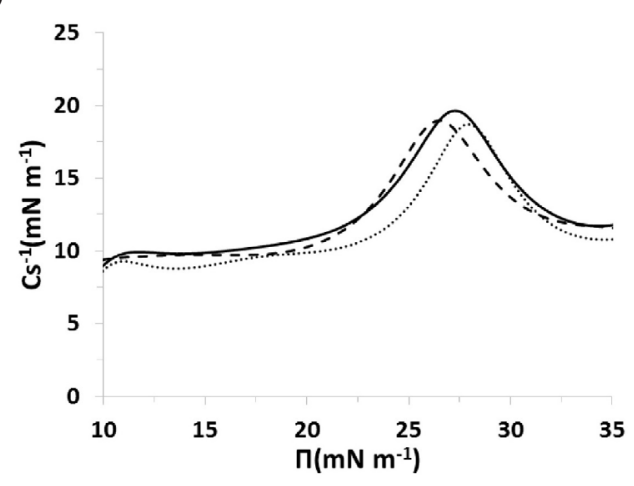

Figure 7. The effect of $\mathrm{pH}$ on the phase behavior of MPP1-lipid monolayers. The compression of MPP1-lipid isotherms in the presence of subphase buffer at pH 6.0 (dotted curve), pH 7.4 (dashed curve), and pH 8.2 (solid curve) (DOPC/SM/Chol (A); DOPS/SM/ $\mathrm{Chol}(\mathbf{B}))$. The compressibility modulus, $\mathrm{Cs}^{-1}-\Pi$ isotherms, was calculated from the corresponding $\Pi-\mathrm{A}$ isotherms as a function of the surface pressure of the films (DOPC/SM/Chol (C); DOPS/SM/ $\mathrm{Chol}(\mathrm{D}))$. E. Maximum compressibility modulus, $\mathrm{Cs}^{-1}{ }_{\max }$, values, expressed as a function of $\mathrm{pH}$ on MPP1-lipid mixtures. DOPC/SM/ Chol (black column) and DOPS/SM/Chol (white dotted bars). The surface area is a relative value for the reason mentioned in the legend of Figure 2. Data represent the mean \pm S.D. $(n=3)$. 
monolayer. A similar mode of action has been described elsewhere, when the interaction and the incorporation of human serum albumin within an octadecylamine monolayer was studied by Yin et al. (2005) and was interpreted as the insertion of human serum albumin molecules into the octadecylamine monolayer.

As reported for other protein-lipid monolayer systems (Krol et al. 2000; Girard-Egrot et al. 2004), the stability of mixed lipid-protein monolayers at high surface pressure, without a significant loss of protein from the monolayer, indicates a strong interaction between the protein and the lipid. Moreover, the symmetric peaks from the $\mathrm{Cs}^{-1}$ curves, and the linear fits provided from Scatchard plots, indicate that a one-step transition process is involved in the MPP1lipid monolayer interaction (Ahluwalia et al. 1991; Dziri et al. 1997; Zhi-Wu Yu et al. 2002).

The addition of MPP1 to the subphase underneath a monolayer of a lipid mixture without cholesterol induced a smaller change in the area at constant surface pressure, compared with that obtained for the same lipid mixture containing cholesterol. This decrease in area may indicate a structural modification, or molecular rearrangement, within the lipid monolayer that is specific to the type of monolayer composition, that is, either the MPP1 can bind directly to cholesterol, as shown by Listowski et al. (2015) or that cholesterol modulates the arrangement of the lipid monolayer, i.e. inducing a phase separation (e.g. Grzybek et al. 2009), which enhances the binding of MPP1. Further studies should help clarify this mechanism.

Our previous simple in silico modelling study on MPP1 (Listowski et al. 2015) showed that this protein contains two hydrophobic/amphipathic stretches of $\sim 12-18$ amino acid residues which could be responsible for the binding or penetration of MPP1 to lipid bi- and mono-layers via hydrophobic interactions. The effect of ionic strength and $\mathrm{pH}$ would shed some light on this issue. However, using low and high ionic-strength or $\mathrm{pH}$ buffers strongly affects the stability of the protein. Therefore, studies in this area were constrained to conditions within which the protein would not be affected and, as a consequence, it was only possible to use rather a low range of ionic strength and $\mathrm{pH}$ values. Using 50, 150 and $250 \mathrm{mM} \mathrm{NaCl}$ did not strongly affect this interaction, but a small minimum of $\mathrm{Cs}^{-1}$ max was observed in presence of $150 \mathrm{mM} \mathrm{NaCl}$ in the subphase, suggesting favourable conditions for the interaction. This was not observed in the case of the lipid monolayer mixture containing DOPS (Fig. 6). Studied range of $\mathrm{pH}$ indicated a small decrease in interaction at $\mathrm{pH} 8.2$ of MPP1 with the studied monolayers in comparison with $\mathrm{pH} 7.4$ and 6.0, respectively (Fig. 7). Overall, it seems that the observed binding may be a result from mixed hydrophobic-hydrophilic protein-lipid interactions which should be a subject of further studies by using other approaches.
Our data using the above techniques demonstrate for the first time that MPP1 binds lipid bi- and monolayers composed of DOPC/SM/Cholesterol in a concentrationdependent manner with $\mathrm{K}_{\mathrm{D}}$ values in the nanomolar range. Furthermore, this interaction is sensitive to the presence of cholesterol in the lipid monolayer. It should be noted that this interaction may, at least in part, explain the participation of MPP1 in resting-state raft organization in erythroid cells

Conflict of interest. The authors declare no conflict of interest.

Acknowledgements. This work was supported by NCN Grant DEC-2012/05/B/NZ1/01638. M.E expresses stupendous gratitude to the effort of the Embassy of Libya in Warsaw, Poland, for financial support. M. E. would also like to express his appreciation to Dr. Joanna Podkalicka for her support, meticulous suggestions, continuous guidance and astute criticism during this project.

\section{References}

Ahluwalia A., De Rossi D., Monici M., Schirone A. (1991): Thermodynamic study of Langmuir antibody films for application to immunosensors. Biosens. Bioelectron. 6, 133-141 https://doi.org/10.1016/0956-5663(91)87037-C

Alloisio N., Dalla Venezia D., Rana A., Andrabi K., Texier P., Gilsanz F., Cartron J. P., Delaunay J., Chishti A. H. (1993): Evidence that red blood cell protein p55 may participate in the skeletonmembrane linkage that involves protein 4.1 and glycophorin C. Blood 82, 1323-1327

Bangham A. D., Standish M. M., Watkins J. C. (1965): Diffusion of univalent ions across the lamellae of swollen phospholipids. J. Mol. Biol. 13, 238-252

https://doi.org/10.1016/S0022-2836(65)80093-6

Bezlyepkina N., Gracià R. S., Shchelokovskyy P., Lipowsky R., Dimova R. (2013): Phase diagram and tie-line determination for the ternary mixture DOPC/eSM/cholesterol. Biophys. J. 104, 1456-1464

https://doi.org/10.1016/j.bpj.2013.02.024

Biernatowska A., Podkalicka J., Majkowski M., HryniewiczJankowska A., Augoff K., Kozak K., Korzeniewski J., Sikorski A. F. (2013): The role of MPP1/p55 and its palmitoylation in resting state raft organization in HEL cells. Biochim. Biophys. Acta - Mol. Cell Res. 1833, 1876-1884

Collado M. I., Go-i F. M., Alonso A., Marsh D. (2005): Domain formation in sphingomyelin/cholesterol mixed membranes studied by spin-label electron spin resonance spectroscopy. Biochemistry 44, 4911-4918 https://doi.org/10.1021/bi0474970

Coste V., Puff N., Lockau D., Quinn P. J., Angelova M. I. (2006): Raft-like domain formation in large unilamellar vesicles probed by the fluorescent phospholipid analogue, C12NBDPC. Biochim. Biophys. Acta 1758, 460-467 https://doi.org/10.1016/j.bbamem.2006.03.003

Devanathan S., Salamon Z., Lindblom G., Grobner G., Tollin G. (2006): Effects of sphingomyelin, cholesterol and zinc ions 
on the binding, insertion and aggregation of the amyloid Abeta1-40 peptide in solid-supported lipid bilayers. FEBS J. 273, 1389-1402 https://doi.org/10.1111/j.1742-4658.2006.05162.x

Dimitratos S. D., Woods D. F., Stathakis D. G., Bryant P. J. (1999): Signaling pathways are focused at specialized regions of the plasma membrane by scaffolding proteins of the MAGUK family. BioEssays 21, 912-921

https://doi.org/10.1002/(SICI)1521-1878(199911)21:11<912::AID-BIES3 >3.0.CO;2-Z

Dziri L., Puppala K., Leblanc R. M. (1997): Surface and spectroscopic properties of acetylcholinesterase monolayer at the air/ water interface. J. Colloid Interface Sci. 194, 37-43 https://doi.org/10.1006/jcis.1997.5069

Engelman D. M. (2005): Introduction membranes are more mosaic than fluid. Nature 438, 578-580 https://doi.org/10.1038/nature04394

Fanning A. S., Anderson J. M. (1996): Protein-protein interactions: PDZ domain networks. Curr. Biol. 6, 1385-1388 https://doi.org/10.1016/S0960-9822(96)00737-3

Frazier M. L., Wright J. R., Pokorny A., Almeida P. F. F. (2007): Investigation of domain formation in sphingomyelin/cholesterol/ POPC mixtures by fluorescence resonance energy transfer and Monte Carlo simulations. Biophys. J. 92, 2422-2433 https://doi.org/10.1529/biophysj.106.100107

Girard-Egrot A., Chauvet J. P., Gillet G., Moradi-Améli M. (2004): Specific interaction of the antiapoptotic protein $\mathrm{Nr}-13$ with phospholipid monolayers is prevented by the $\mathrm{BH} 3$ domain of Bax. J. Mol. Biol. 335, 321-231 https://doi.org/10.1016/j.jmb.2003.10.028

Gosens I., Wijk E., Kersten F. F. J., Krieger E., Zwaag B., Marker T., Letteboer S. J. F., Dusseljee S., Peters T., Spierenburg H. A. et al. (2007): MPP1 links the Usher protein network and the Crumbs protein complex in the retina. Hum. Mol. Genet. 16, 1993-2003 https://doi.org/10.1093/hmg/ddm147

Grzybek M., Kubiak J., Łach A., Przybyło M., Sikorski A. F. (2009): A raft-associated species of phosphatidylethanolamine interacts with cholesterol comparably to sphingomyelin. A LangmuirBlodgett monolayer study. PLoS One 4, e5053. https://doi.org/10.1371/journal.pone.0005053

Holopainen J. M., Subramanian M., Kinnunen P. K. (1998): Sphingomyelinase induces lipid microdomain formation in a fluid phosphatidylcholine/sphingomyelin membrane. Biochemistry 37, 17562-17570 https://doi.org/10.1021/bi980915e

Krol S., Ross M., Sieber M., Künneke S., Galla H. J., Janshoff A. (2000): Formation of three-dimensional protein-lipid aggregates in monolayer films induced by surfactant protein B. Biophys. J. 79, 904-918 https://doi.org/10.1016/S0006-3495(00)76346-6

Kulma M., Herec M., Grudzilski W., Anderluh G., Gruszecki W. I., Kwiatkowska K., Sobota A. (2010): Sphingomyelin-rich domains are sites of lysenin oligomerization: Implications for raft studies. Biochim. Biophys. Acta 1798, 471-481 https://doi.org/10.1016/j.bbamem.2009.12.004

Łach A., Grzybek M., Heger E., Korycka J., Wolny M., Kubiak J., Kolondra A., Boguslawska D. M., Augoff K., Majkowski M. et al. (2012): Palmitoylation of MPP1 (Membrane-palmitoylated Protein 1)/p55 is crucial for lateral membrane organization in erythroid cells. J. Biol. Chem. 287, 18974-18984

https://doi.org/10.1074/jbc.M111.332981

Lehtonen J. Y., Holopainen J. M., Kinnunen P. K. (1996): Evidence for the formation of microdomains in liquid crystalline large unilamellar vesicles caused by hydrophobic mismatch of the constituent phospholipids. Biophys. J. 70, 1753-1760 https://doi.org/10.1016/S0006-3495(96)79738-2

Listowski M. A., Leluk J., Kraszewski S., Sikorski A. F. (2015): Cholesterol interaction with the MAGUK protein family member, MPP1, via CRAC and CRAC-Like Motifs: An in silico docking analysis. PLoS One 10, e0133141 https://doi.org/10.1371/journal.pone.0133141

Marchenkova M. A., Dyakova Y. A., Tereschenko E. Y., Kovalchuk M. V., Vladimirov Y. A. (2015): Cytochrome c complexes with cardiolipin monolayer formed under different surface pressure. Langmuir 31, 12426-12436 https://doi.org/10.1021/acs.langmuir.5b03155

Mburu P., Kikkawa Y., Townsend S., Romero R., Yonekawa H., Brown S. D. M. (2006): Whirlin complexes with p55 at the stereocilia tip during hair cell development. Proc. Natl. Acad. Sci. USA 103, 10973-10978 https://doi.org/10.1073/pnas.0600923103

Morton L. A., Saludes J. P., Yin H. (2012): Constant pressurecontrolled extrusion method for the preparation of nano-sized lipid vesicles. J. Vis. Exp. 64, 4151 https://doi.org/10.3791/4151

Mouritsen O. G. (1991): Theoretical models of phospholipid phase transitions. Chem. Phys. Lipids 57, 179-194 https://doi.org/10.1016/0009-3084(91)90075-M

Mouritsen O. G., Bloom M. (1984): Mattress model of lipid-protein interactions in membranes. Biophys. J. 46, 141-153 https://doi.org/10.1016/S0006-3495(84)84007-2

Nunomura W., Takakuwa Y., Parra M., Conboy J., Mohandas N. (2000): Regulation of protein 4.1R, p55, and glycophorin C ternary complex in human erythrocyte membrane. J. Biol. Chem. 275, 24540-24546 https://doi.org/10.1074/jbc.M002492200

Nyholm T. K. M., Lindroos D., Westerlund B., Slotte J. P. (2011): Construction of a DOPC/PSM/cholesterol phase diagram based on the fluorescence properties of trans -parinaric acid. Langmuir 27, 8339-8350

https://doi.org/10.1021/la201427w

Podkalicka J., Biernatowska A., Majkowski M., Grzybek M., Sikorski A. F. (2015): MPP1 as a factor regulating phase separation in giant plasma membrane-derived vesicles. Biophys. J. 108, 2201-2211 https://doi.org/10.1016/j.bpj.2015.03.017

Rouser G., Siakotos A. N., Fleischer S. (1966): Quantitative analysis of phospholipids by thin-layer chromatography and phosphorus analysis of spots. Lipids 1, 85-86 https://doi.org/10.1007/BF02668129

Ruff P., Speicher D. W., Husain-Chishti A. (1991): Molecular identification of a major palmitoylated erythrocyte membrane protein containing the src homology 3 motif. Proc. Natl. Acad. Sci. USA 88, 6595-6599 https://doi.org/10.1073/pnas.88.15.6595 
Rytömaa M., Kinnunen P. K. (1996): Dissociation of cytochrome c from liposomes by histone H1. Comparison with basic peptides. Biochemistry 35, 4529-4539 https://doi.org/10.1021/bi952413w

Seo P.-S., Jeong J. J., Zeng L., Takoudis C. G., Quinn B. J., Khan A. A., Hanada T., Chishti A. H. (2009): Alternatively spliced exon 5 of the FERM domain of protein 4.1R encodes a novel binding site for erythrocyte p55 and is critical for membrane targeting in epithelial cells. Biochim. Biophys. Acta 1793, 281-289 https://doi.org/10.1016/j.bbamcr.2008.09.012

Söderlund T., Jutila A., Kinnunen P. K. (1999): Binding of adriamycin to liposomes as a probe for membrane lateral organization. Biophys. J. 76, 896-907 https://doi.org/10.1016/S0006-3495(99)77253-X

Tsukamoto M., Kuroda K., Ramamoorthy A., Yasuhara K. (2014) Modulation of raft domains in a lipid bilayer by boundaryactive curcumin. Chem. Commun. (Camb). 50, 3427-2430 https://doi.org/10.1039/c3cc47738j

Wilkins M. R., Gasteiger E., Bairoch A., Sanchez J. C., Williams K. L., Appel R. D., Hochstrasser D. F. (1999): Protein identification and analysis tools in the ExPASy server. Methods Mol. Biol. 112, 531-552
Yu L., Guo L., Ding J. L., Ho B., shen Feng S., Popplewell J., Swann M., Wohland T. (2009): Interaction of an artificial antimicrobial peptide with lipid membranes. Biochim. Biophys. Acta 1788, 333-344

Yuan C., Furlong J., Burgos P., Johnston L. J. (2002): The size of lipid rafts: an atomic force microscopy study of ganglioside GM1 domains in sphingomyelin/DOPC/cholesterol membranes. Biophys. J. 82, 2526-2535 https://doi.org/10.1016/S0006-3495(02)75596-3

Yin F., Kafi A. K. M., Shin H. K., Kwon Y. S. (2005): Human serum albumin-octadecylamine Langmuir-Blodgett film formed by spreading human serum albumin solution directly on subphase's interface covered with a layer of octadecylamine. Thin Solid Films 488, 223-229

https://doi.org/10.1016/j.tsf.2005.04.030

Zhi-Wu Y., Jin J., Cao Y. (2002): Characterization of the liquidexpanded to liquid-condensed phase transition of monolayers by means of compressibility. Langmuir 18, 4530-4531 https://doi.org/10.1021/la011840+

Received: November 14, 2016

Final version accepted: January 12, 2017

First published online: June 27, 2017 